\title{
A STUDY OF LINEAR TIME-VARYING SYSTEMS SUBJECT TO STOCHASTIC DISTURBANCES
}

\author{
S. Y. Chan and K. Chuang* \\ Electrical Engineering Department, The University of Michigan, Ann Arbor, Michigan, U.S.A.
}

\begin{abstract}
Summary-This paper is concerned with the analysis, in a stochastic sense, of systems described by linear differential equations with random disturbances, which often arise in the study of the variational behavior of an optimal control system along its nominal trajectory due to random disturbances in plant parameters or measuring errors in state variables.

The random vector may be a white noise vector or may be generated by differential equations excited by white noise. By means of the Fokker-Planck equation the general result not only reveals the stability property of the system but also enables one to determine the state of the system at every instant of time in a stochastic sense. Experimental verification is given by simulating a second order system on an analog computer and the result is found to be in agreement with theory.
\end{abstract}

\section{INTRODUCTION}

THIS PAPER describes a method of analyzing the behavior of time-varying systems under the influence of stochastic disturbances. By means of the known property of state transition matrix a close-form solution for the probability density function for the given system can be obtained.

During the past 15 years there has been much interest in analyzing the behavior of automatic control systems under the influence of random disturbances. If the system is linear and the output-input relationship is governed by a linear differential equation with constant coefficients, then the well-known techniques using transfer function, correlation function, etc., are adequate to analyze the behavior of the system. In the case that the behavior of a system is described by a set of linear differential equations with constant coefficients under the influence of random disturbances, with practically white spectra the method of Fokker-Planck equation was used by WANG and UHLENBECK [1].

The present paper is primarily concerned with the analysis of linear time-varying systems excited by white noise and to a greater extent, of linear time-varying systems under the influence of stochastic processes which are non-white as well as non-stationary. However, the assumption on these processes is that they are generated by a set of linear differential equations excited by white noise; this being frequently the most realistic way of viewing the operation of physical systems. A white noise random process can be considered only as a limiting case which will never occur in practice. The most common examples of stationary process with rational power spectral density, especially in noise applications to electric circuits, are those which can be considered as the spectral density of the output from a time invariant lumped-parameter linear circuit excited by white noise.

* The work reported in this paper was supported by National Science Foundation/Grant GP-1381.

[1] M. C. Wang and G. E. Uhlendeck: On the theory of Brownian motion II, Rev. Mod. Phys. 17, Nos. 2 and 3, April-July (1945). 


\section{PROBABILISTIC CHARACTERIZATION OF A MARKOV PROCESS}

Let $P(t, x / \tau, y) \mathrm{d} y$ be the conditional probability that at time $\tau$ the random vector $\xi(\tau)=\left(\xi_{1}(\tau), \ldots, \xi_{n}(\tau)\right) \varepsilon[y, y+\mathrm{d} y]$ under the condition that at $t, \xi(t)=x$. Then a Markov process can be defined by stating that for such a process the conditional probability $P(t, x / \tau, y) \mathrm{d} y$ that the random vector $\xi(\tau)=\left(\xi_{1}(\tau), \ldots, \xi_{0}(\tau)\right)$ assumes the values in $\left[y_{i}, y_{i}+\mathrm{d} y_{i}\right](i=1, \ldots, n)$ at time $\tau$ depends besides on $y_{i}$ and $\tau$ only on the vector $\xi$ at the previous time $t$. Any additional information concerning the states of the system at instants of time earlier than $t$ for the process has no effect on the function $P(t, x / \tau, y)$. In other words, a Markov process is completely described by the conditional probability density frunction $P(t, x / \tau, y)$. It can be shown that such a process will always satisfy the generalized Markov equation, i.e. for the instants of time $t, s, \tau$ such that $t<s<\tau$

$$
P(t, x / \tau, y)=\int P(t, x / s, z) P(s, z / \tau, y) \mathrm{d} z
$$

It is assumed in what follows that any integration is to be carried out over the entire $n$-dimensional state space $R_{n}$ unless the limits of integration are specified otherwise.

\section{THE FOKKER-PLANCK EQUATION}

In order to use the Fokker-Planck equation for the analysis of physical systems under stochastic disturbances we need the following assumptions:

(a) Condition of strong continuity, i.e.

$$
\lim _{\Delta t \rightarrow 0} \frac{1}{\Delta t} \int_{|y-x| \geq \delta} P(t-\Delta t, x / t, y) \mathrm{d} y=0
$$

for every positive number $\delta$. The quantity $|y-x|$ can be considered as the Euclidean length in $R_{n}$.

(b) for any $\delta>0$, the limits

$$
\begin{aligned}
& \lim _{\Delta t \rightarrow 0} \frac{1}{\Delta t} \int_{|y-x|<\delta}\left(y_{i}-x_{i}\right) P(t-\Delta t, x / t, y) \mathrm{d} y=a_{i}(t, x) \quad i=1, \ldots, n \\
& \lim _{\Delta t \rightarrow 0} \frac{1}{\Delta t} \int_{|y-x|<\delta}\left(y_{i}-x_{i}\right)\left(y_{j}-x_{j}\right) P(-\Delta t, x / t, y) \mathrm{d} y=b_{i j}(t, x) \quad i, j=1, \ldots n
\end{aligned}
$$

exist and the convergence of (3) and (4) is uniform in $x$.

(c) the partial derivatives

$$
\frac{\partial P(t, x / \tau, y)}{\partial \tau}, \frac{\partial}{\partial y_{i}}\left[a_{i}(\tau, y) P(t, x / \tau, y)\right] \frac{\partial^{2}}{\partial y_{i} \partial y_{j}}\left[b_{i j}(\tau, y) P(t, x / \tau, y)\right]
$$

exist and are continuous for all $t, x, \tau>t$ and $y$.

Under the assumptions (1) through (5) it can be readily shown the conditional probability density function satisfies the Fokker-Planck equation

$$
\begin{aligned}
\frac{\partial P(t, x / \tau, y)}{\partial \tau}= & -\sum_{i=1}^{n} \frac{\partial}{\partial y_{i}}\left[a_{i}(\tau, y) P(t, x / \tau, y)\right] \\
& +\frac{1}{2} \sum_{i, j=1}^{n} \frac{\partial^{2}}{\partial y_{i} \partial y_{j}}\left[b_{i j}(\tau, y) P(t, x / \tau, y)\right]
\end{aligned}
$$


4. LINEAR TIME-VARYING SYSTEM EXCITED BY WHITE NOISE

Let us consider a linear time-varying system of the form

$$
\frac{\mathrm{d} z_{i}}{\mathrm{~d} t}=\sum_{k=1}^{n} C_{i k}(t) z_{k}+\sum \mathrm{d}_{i k}(t) n_{k}(t), \quad z_{i}(0)=z_{0 i}, \quad i=1, \ldots, n
$$

Equations (7) can be put into vector form

$$
\frac{\mathrm{d} z}{\mathrm{~d} t}=C(t) z+D(t) n(t) \quad z(0)=z_{0}
$$

The elements $C_{i k}(t)$ and $C_{i k}(t)$ are continuous functions of $t \geq 0$ and $n_{k}(t)$ are the components of a random noise vector $n(t)=\left(n_{1}(t), \ldots, n_{n}(t)\right.$, satisfying the following conditions:

(a) Each component $n_{i}(i=1, \ldots, n)$ is a Gaussian white noise with zero mean and a constant spectral density of $4 D_{i}$, or

and

$$
E\left[n_{i}(t)\right]=0
$$

$$
E\left[n_{i}\left(t_{1}\right) n_{i}\left(t_{2}\right)\right]=2 D_{i} \delta\left(t_{1}-t_{2}\right),
$$

where $\delta\left(t_{1}-t_{2}\right)$ is the Dirac singular function.

(b) The components $n_{i}(t)$ and $n_{j}(t), i \neq j$, are uncorrelated so that

$$
E\left[n_{i}\left(t_{1}\right) n_{j}\left(t_{2}\right)\right]=0, \quad i, j=1, \ldots, n
$$

With the above assumptions, the Fokker-Planck equation (6) associated with the system (7) reduces to the following form:

where

$$
\frac{\partial P}{\partial t}=-\sum_{i, k=1}^{n} \frac{\partial}{\partial z_{i}}\left[C_{i k}(t) z_{k} P\right]+\sum_{i, j=1}^{n} f_{i}(t) \frac{\partial^{2} P}{\partial z_{i} \partial z_{j}},
$$

$$
f_{i j}(t)=\sum_{k=1}^{n} D_{k} \mathrm{~d}_{i k}(t) \mathrm{d}_{j k}(t)
$$

At $t=0$, the initial condition on $P$ is given by

$$
\left.P\right|_{t=0}=\prod_{i=1}^{n} \delta\left(z_{i}-z_{0 i}\right)
$$

and $P \rightarrow 0$ as $z_{i} \rightarrow \pm \infty$. The solution of (11) which satisfies the above initial and boundary conditions is called the fundamental solution of the Fokker-Planck equation associated with system (7).

The fundamental solution, that is, the conditional probability of the system (7), is provided by the following theorem:

Theorem 1. For the given system (7) satisfying conditions (9) and (10), the fundamental solution, $P$, of the associated Fokker-Planck equation (11) is given by

$$
P\left(0, z_{0} / t, z\right)=\frac{1}{(2 \pi)^{n / 2}|\lambda|^{1 / 2}} \exp \left[-\frac{1}{|\lambda|} \sum_{i, j=1}^{n}|\lambda|_{i j}\left(z_{i}-m_{i}\right)\left(z_{j}-m_{j}\right)\right]
$$

where $|\lambda|_{i j}$ is the cofactor of the elements $\lambda_{i j}$ in the determinant $|\lambda|$ of the covariance matrix 


$$
\lambda=\left[\begin{array}{cccc}
\lambda_{11} & \lambda_{12} & \ldots & \lambda_{1 n} \\
\lambda_{21} & \lambda_{22} & \ldots & \lambda_{2 n} \\
\cdot & \cdot & . & \cdot \\
\lambda_{n 1} & \lambda_{n 2} & \ldots & \lambda_{n n}
\end{array}\right]
$$

in which the elements $\lambda_{i j}$ and $m_{i}$ are given, respectively, by

$$
\begin{gathered}
\lambda_{i j}(t)=2 \sum_{i, j=1}^{n} \phi_{k r}(t) \phi_{l s}(t) \int_{0}^{t} f_{i j}(t) \psi_{i k}(t) \psi_{j}(t) \mathrm{d} t . \\
m_{i}(t)=\sum_{k=1}^{n} \phi_{k j}(t) z_{0 k} .
\end{gathered}
$$

$\phi_{i j}(t)$ and $\psi_{i j}(t)$ are the elements of the matrices $\Phi$ and $\Psi$ satisfying the following equations

$$
\begin{array}{ll}
\dot{\Phi}^{\prime}=C(t) \Phi^{\prime}, & \Phi^{\prime}(0)=I \\
\dot{\Psi}=-C^{\prime}(t) \Psi, & \Psi(0)=I
\end{array}
$$

where $\Phi^{\prime}$ and $C^{\prime}(t)$ are the transposes of the matrices $\dot{\Phi}$ and $C(t)$ respectively and $I$ is the identity matrix.

Proof. To find the fundamental solution of (11) with the initial condition (13) we shall generalize the method of WANG and UHLENBECK [1] which was first used in the theory of Brownian motion with Brownian particles governed by a set of linear differential equations with constant coefficients in which the characteristic roots were assumed to be real and distinct. Let us define the Fourier transform of the conditional probability density function $P$ by

$$
g(\xi, t)=\int \mathrm{d} z P \exp \left[-i \sum_{k=1}^{n} \xi_{k} z_{k}\right]=\int_{-\infty}^{\infty} \ldots \int \mathrm{d} z_{1} \ldots \mathrm{d} z_{n} P \exp \left[-i \sum_{k=1}^{n} \xi_{k} z_{k}\right]
$$

where $\xi=\left(\xi_{1}, \ldots, \xi_{n}\right)$.

Taking the Fourier transform of both sides of (11) and integrating by parts with respect to $z_{i}$ and $z_{j}$ we find

$$
\frac{\partial g(\xi, t)}{\partial t}=\sum_{i, j=1}^{n} C_{i j}(t) \xi_{i} \frac{\partial g(\xi, t)}{\partial \xi_{j}}-g(\xi, t) \sum_{i, j=1}^{n} f_{i j}(t) \xi_{i} \xi_{j}
$$

Equation (21) is a linear first order partial differential equation and its solution can be obtained by the method of characteristics [2]. The auxiliary system of equations is of the following form:

$$
\frac{\mathrm{d} t}{1}=\frac{-\mathrm{d} \xi k}{\sum_{i=1}^{n} C_{i k}(t) \xi_{i}}=\frac{-\mathrm{d} g}{g \sum_{i, j=1}^{m} f_{i j}(t) \xi_{i} \xi_{j}} \quad k=1, \ldots, n
$$

Writing equation (22) in matrix form, we have,

$$
\begin{aligned}
& \dot{\xi}=-C^{\prime}(t) \xi, \\
& \dot{g}=-g \sum_{i, j=1}^{n} f_{i j}(t) \xi_{i} \xi_{j}
\end{aligned}
$$

[2] L. E. Etcolts: Differential Equations, p. 298. Gordon and Breach, Delhi (1961). 
It can be seen that the first equation of (23) is the adjoint equation of the original system (8).

Let $\psi(t)$ be the state transition matrix of the first equation of (23), i.e. $\psi(t)$ is the solution of

$$
\psi(t)=-C^{\prime}(t) \psi(t), \quad \psi(0)=I .
$$

Then the solution of the first equation of (23) is given by

$$
\xi(t)=\psi(t) \xi_{0}
$$

or in terms of components

$$
\xi_{i}(t)=\sum_{j=1}^{n} \psi_{i j}(t) \xi_{0 j}, \quad i=1, \ldots, n
$$

where $\psi_{i j}$ if the $i j^{\text {th }}$ element of the matrix $\psi$, and $\xi_{0 j}$ is the $j^{\text {th }}$ component of an arbitrary initial vector $\xi_{0}$. By the property of nonsingularity of state transition matrix [3] we find from (25)

or

$$
\xi_{0}=\psi^{-1}(t) \xi(t)=\Phi(t) \xi(t)
$$

$$
\xi_{0 i}=\sum_{j=1}^{n} \phi_{i j}(t) \xi_{j}(t), \quad i=1, \ldots, n
$$

where $\phi_{i j}(t)$ is the $i j^{\text {th }}$ element of $\Phi(t)$, which is the transpose of the state transition matrix of the system (8).

The solution of the second equation of (23) can be found as

$$
g=g_{0} \exp \left\{-\sum_{i, j=1}^{m} \int_{0}^{t} f_{i j}(t) \xi_{i}(t) \xi_{j}(t) \mathrm{d} t\right\}
$$

Using relations (26) and (28), equation (29) reduces to

$$
g=g_{0} \exp \left\{-\sum_{\substack{i, j, k=1 \\ i, r, s}}^{n} \phi_{k r}(t) \phi_{l s}(t) \xi_{r} \xi_{s} \int_{0}^{t} f_{i j}(t) \psi_{i k}(t) \psi_{j l}(t) \mathrm{d} t\right\}
$$

The general solution of (21) is of the form

$$
\Gamma\left(\xi_{01}, \ldots, \xi_{0 n}, g_{0}\right)=0
$$

where $\Gamma$ is an arbitrary function. Making use of (28), (30) and (31) the solution of (21) reduces to

$$
g(\xi, t)=F\left[\sum_{j=1}^{n} \phi_{1 j}(t) \xi_{j} \ldots, \sum_{j=1}^{n} \phi_{n j}(t) \xi_{j}, t\right] \exp \left[-\sum_{r, s=1}^{n} K_{r s}(t) \xi_{r} \xi_{s}\right],
$$

where

$$
K_{r s}(t) \Delta \sum_{\substack{i, j, j=1 \\ k, l}}^{n} \phi_{k r}(t) \phi_{l s}(t) \int_{0}^{t} f_{i j}(t) \psi_{l k}(t) \psi_{j l}(t) \mathrm{d} t
$$

[3] L. A. ZADEH and C. A. Desoer: Linear System Theory, p. 344. McGraw-Hill, New York (1963). 
and $F$ is another arbitrary function. Making use of the initial condition (13) together with (20) and (32), the fundamental solution of (21) becomes

$$
g(\xi, t)=\exp \left\{-i \sum_{k, i=1}^{n} \phi_{k j}(t) \xi_{j} z_{0 k}-\sum_{r, s=1}^{n} K_{r s}(t) \xi_{r} \xi_{s}\right\}
$$

It can be easily verified that $g(\xi, t)$ is actually the solution of (21). Let us define

$$
m_{j}(t) \Delta \sum_{k=1}^{n} \phi_{k j}(t) z_{0 k} \quad j=1, \ldots, n
$$

and

$$
\lambda_{r s}(t) \Delta 2 K_{r s}(t)=2 \sum_{\substack{l, k \\ k, l}=1}^{n} \phi_{k r}(t) \phi_{l s}(t) \int_{0}^{t} f_{l j}(t) \psi_{l k}(t) \psi_{j l}(t) \mathrm{d} t, \quad r, s=1, \ldots, n
$$

then equation (34) reduces to

$$
g(\xi, t)=\exp \left\{-i \sum_{j=1}^{n} \xi_{j} m_{j}(t)-\frac{1}{2} \sum_{r, s=1}^{n} \lambda_{r s}(t) \xi_{r} \xi_{s}\right\}
$$

Equation (37) is simply the Fourier transform of an $n$-dimensional distribution with means and variances expressed respectively by (35) and (36) with the corresponding probability density function [4].

$$
P\left(0, z_{0} / t, z\right)=\frac{1}{(2 \pi)^{n / 2}|\lambda|^{1 / 2}} \exp \left[-\frac{1}{2|\lambda|} \sum_{i, j=1}^{n}|\lambda|_{i j}\left(z_{i}-m_{i}\right)\left(z_{j}-m_{j}\right)\right]
$$

which is what we want to prove.

If the initial position of the system (7) or (8) is known exactly then the initial probability density function $P\left(0, z_{0}\right)$ can be written as

$$
P\left(0, z_{0}\right)=\delta\left(z_{0}-a_{0}\right)=\prod_{i=1}^{n} \delta\left(z_{0 t}-a_{0 i}\right),
$$

and the probability density function $P(t, z)$ will be the same as equation (38) with means and variances given by (35) and (36) with $z_{0 k}$ in (35) replaced by $a_{0 k}$.

However, if only the initial distribution $P\left(0, z_{0}\right)$ is known, then the probability density function $P(t, z)$ is given in the form of an integral

$$
P(t, z)=\int P\left(0, z_{0}\right) P\left(0, z_{0} / t, z\right) \mathrm{d} z_{0}
$$

[4] W. B. DayenPort, Jr. and W. L. Root: Random Signals and Noise, p. 152. McGraw-Hill, New York (1958). 
Knowing the probability density distribution $P(t, z)$ the expected values as well as the moments of the solution of the given system can be determined at every instant of time. This is true even though the initial position of the system is not exactly known, but is only given by its probability distribution. This, of course, determines the stability of the system in a stochastic sense.

\section{LINEAR TIME-VARYING SYSTEM WITH DISTURBANCES GENERATED BY DIFFERENTIAL EQUATIONS EXCITED BY WHITE NOISE}

In the previous section we assumed that random disturbances $n(t)$ of the linear timevarying system were caused by white noise. A white noise random process can be considered only as a limiting case which will never occur in practice.

For the above reason the natural generalization is to assume that the random noise vector $n(t)=\left(n_{1}(t), \ldots, n_{n}(t)\right)$ in the system (8) is governed by the following set of linear differential equations

$$
\frac{\mathrm{d}^{\alpha_{i}} n_{i}}{\mathrm{~d} t^{\alpha_{i}}}+\beta(t) i\left(\alpha_{i}-1\right) \frac{d^{\alpha_{i}-1} n_{i}}{\mathrm{~d} t^{\alpha_{i}-1}}+\ldots+\beta_{i 1}(t) \frac{\mathrm{d} n_{i}}{\mathrm{~d} t}+\beta_{10}(t) n_{i}=w_{i} \quad i=1, \ldots, n
$$

where $w_{i}$ is the $i^{\text {th }}$ component of the white noise vector $w(t)=\left[w_{1}(t), \ldots, w_{n}(t)\right]$.

For each $i$, equation (41) can be transformed into a set of first order linear differential equations. Let

and

$$
z_{j}=v_{j} \quad j=1, \ldots, n
$$

$$
n_{i}=v_{n+a_{1}}+\ldots+a_{i-1}+1, \quad i=1, \ldots, n
$$

then equation (41) can be written into the following form

$$
\begin{gathered}
\frac{\mathrm{d} v_{n+\alpha_{1}+\ldots+\alpha_{i-1}+1}}{\mathrm{~d} t}=v_{n+\alpha_{1}}+\ldots+\alpha_{i-1}+2 \\
\frac{\mathrm{d} v_{n+\alpha_{1}+\ldots+\alpha_{i-1}+2}}{\mathrm{~d} t}=v_{n+\alpha_{1}+\ldots+\alpha_{i-1}+3} \\
\frac{\mathrm{d} v_{n+\alpha_{1}}+\ldots+\alpha_{i}}{\mathrm{~d} t}=-\beta_{i\left(\alpha_{i}-1\right)}(t) v_{n+\alpha_{1}}+\ldots+\alpha_{i}-\ldots-\beta_{i 0}(t) v_{n+\alpha_{1}}+\ldots+\alpha_{i-1}+1+w_{i} \\
i=1, \ldots, n
\end{gathered}
$$

Adjoining equations (43) to the linear system (7)

$$
\frac{\mathrm{d} z_{i}}{\mathrm{~d} t}=\sum_{k=1}^{n} C_{i k}(t) z_{k}+\sum_{k=1}^{n} \mathrm{~d}_{i k}(t) n_{k}(t) \quad i=1, \ldots, n
$$


one obtains a set of linear first order differential equations in the expanded state space of dimension $N \Delta n+\alpha_{1}+\ldots+\alpha_{n}$ excited by white noise.

In terms of components of state vector $\left(v_{1}, \ldots, v_{N}\right)$ in the expanded space we have

$$
\begin{aligned}
& \frac{\mathrm{d} v_{1}}{\mathrm{~d} t}=C_{11}(t) v_{1}+\ldots+C_{1 n}(t) v_{n}+d_{11}(t) v_{n+1}+d_{12}(t) v_{n+a_{1}+1}+\ldots \\
& +d_{1 n}(t) v_{n+a_{1}}+\ldots+a_{n-1}+1 \\
& \frac{\mathrm{d} v_{n}}{\mathrm{~d} t}=C_{n l}(t) v_{1}+\ldots+C_{n n}(t) v_{n}+d_{n t}(t) v_{n+1}+d_{n 2}(t) v_{n+\alpha_{1}+1}+\ldots \\
& \frac{\mathrm{d} v_{n+1}}{\mathrm{~d} t}=v_{n+2} \\
& +d_{n n}(t) v_{n+\alpha_{1}}+\ldots+\alpha_{n-1}+1 \\
& \frac{\mathrm{d} v_{n+\alpha}}{\mathrm{d} t}=\beta_{1\left(\alpha_{1}-1\right)}(t) v_{n+\alpha_{1}}-\ldots-\beta_{10}(t) v_{n+1}+F_{n+\alpha_{1}}(t) \\
& \frac{\mathrm{d} v_{n+a_{1}+1}}{\mathrm{~d} t}=v_{n+a_{1}+2} \\
& \frac{\mathrm{d} v_{n+\alpha_{1}+\alpha_{2}}}{\mathrm{~d} t}=-\beta_{2\left(\alpha_{2}-1\right)}(t) v_{n+\alpha_{1}+\alpha_{2}}-\ldots-\beta_{20}(t) v_{n+\alpha_{1}+1}+F_{n+\alpha_{1}+\alpha_{2}}(t) \\
& \frac{\mathrm{d} v_{n+\alpha_{1}}+\ldots+a_{n-1}+1}{\mathrm{~d} t}=v_{n+\alpha_{1}}+\ldots+\alpha_{n-1}+2 \\
& \frac{\mathrm{d} v_{n+\alpha_{1}}+\ldots+\alpha_{n}}{\mathrm{~d} t}=-\beta_{n\left(\alpha_{n}-1\right)}(t) v_{n+\alpha_{1}}+\ldots a_{n}-\ldots \\
& -\beta_{n 0}(t) v_{n+a_{1}}+\ldots+\alpha_{n-1}+1+F_{n+a_{1}}+\ldots+\alpha_{n}(t)
\end{aligned}
$$

Writing equation (44) in matrix form we obtain

$$
\frac{\mathrm{d} v}{\mathrm{~d} t}=E(t) v+F(t)
$$


高

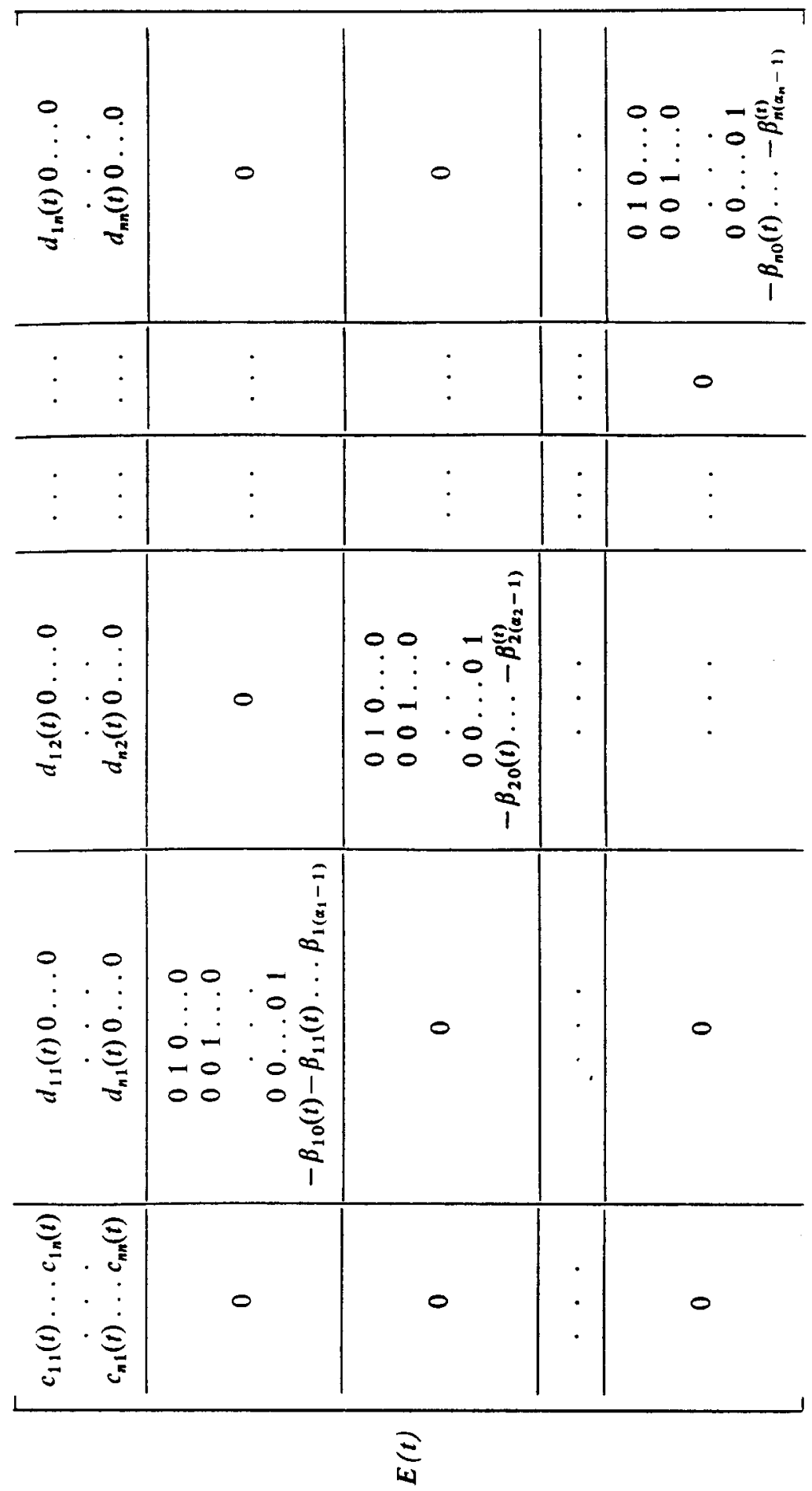


and $F(t)$ is a column matrix in which the non zero elements are given by

$$
F n+\sum_{j=1}^{i} \alpha_{j}(t)=w_{i}(t) \quad i=1, \ldots, n
$$

It is readily seen that the expanded system (45) is again a linear system excited by white noise and is in essence the same as equation (8). Therefore, we can summarize the above results into the following.

Theorem 2. For the given system described by equations (8) and (41) with the white noise vector $w(t)$ satisfying conditions (9) and (10) the fundamental solution, $P\left(0, z_{0} / t, z\right)$, of the associated Fokker-Planck equation in the expanded state space of dimension $N$ is again given by the expression (11) satisfying conditions (13) through (17) with $n, C(t), D(t)$ and $n(t)$ replaced, respectively, by $N, E(t), I$, and $F(t)$.

The results developed in this paper can be applied to a wide class of control systems such as for the determination of average changes in trajectory and the performance index of optimal control systems due to random disturbances in plant parameters or noisy observation of state variables [5].

\section{EXAMPLE}

Let us consider an optimal control system which can be considered as a single axis model of a vehicle with a reaction wheel [6]. The control problem is to transfer the vehicle from some fixed initial attitude to some fixed final attitude and the control voltage will be chosen such that the energy delivered to the servomotor is minimum. The equation of motion can be described by the following differential equations

$$
\begin{aligned}
& \frac{d x_{1}}{d \tau}=x_{2} \\
& \frac{d x_{2}}{d \tau}=-x_{2}+u(\tau)
\end{aligned}
$$

with the initial conditions, $x_{1}(0)=x_{10}, x_{2}(0)=0$

The control problem is to find $u(\tau)$ such that it transfers the vehicle from the initial state $\left(x_{10}, 0\right)$ to the final state $(0,0)$ and at the same time it minimizes the functional

$$
J=\int_{0}^{T} u\left(u-x_{2}\right) \mathrm{d} \tau
$$

for a given transition time $T$.

It can be shown that the energy (49) is minimized if the control function $u(\tau)$ assumes the following form

$$
u\left(x_{1}, x_{2}\right)=x_{2}+\frac{36}{T^{3}} x_{10}\left[\frac{2 x_{1}-x_{10}}{2 x_{2}-(6 / T) x_{10}}\right]
$$

[5] S. Y. Chan: Analysis of variations in optimal control systems subject to deterministic and stochastic disturbances. Ph.D. Thesis, The University of Michigan (1965).

[6] I. FlUGGe-Lotz and H. MARBACH: The optimal control of some attitude control systems for different performance criteria, 12-1 (1962). Joint Automatic Control Conference, New York. 
Substituting (50) into (48) gives

$$
\begin{aligned}
& \dot{x}_{1}=x_{2} \\
& \dot{x}_{2}=\frac{36}{T^{3}} x_{10}\left[\frac{2 x_{1}-x_{10}}{2 x_{2}-(6 / T) x_{10}}\right]
\end{aligned}
$$

If we let

$$
\begin{aligned}
x_{1}(0) & =20 \\
x_{2}(0) & =0 \\
x_{1}(T) & =0 \\
x_{2}(T) & =0 \\
T & =10
\end{aligned}
$$

then equation $(51)$ reduces to

$$
\begin{aligned}
& \dot{x}_{1}=x_{2} \\
& \dot{x}_{2}=0.72\left[\frac{x_{1}-10}{x_{2}-6}\right] .
\end{aligned}
$$

The solution of (52) gives the optimal trajectory.

If there is random noise $n_{1}$ in the optimal control problem, the equations of motion will be

$$
\begin{aligned}
& \dot{y}_{1}=y_{2} \\
& \dot{y}_{2}=0.72\left[\frac{y_{1}+n_{1}-10}{y_{2}-6}\right]
\end{aligned}
$$

where $y$ 's are the components of the actual trajectory of the system.

Let us assume that the initial values of the system (53) are given by

$$
\begin{aligned}
& y_{1}(0)=18 \\
& y_{2}(0)=0,
\end{aligned}
$$

then by combining equations (52) and (53) the equations of first variation along the optimal trajectory due to the presence of noise $n_{1}$ become

$$
\begin{aligned}
& \dot{z}_{1}=z_{2} \\
& \dot{z}_{2}=0.72\left[\frac{1}{x_{2}-6}\left(z_{1}+n_{1}\right)-\frac{x_{1}-10}{\left(x_{2}-6\right)^{2}} z_{2}\right]
\end{aligned}
$$

with the initial conditions $z_{1}(0)=-2$ and $z_{2}(0)=0$, where $z_{1}=y_{1}-x_{1}$ and $z_{2}=y_{2}-x_{2}$. Equation (54) can be written in the following vector form,

$$
\dot{z}=C(\tau) z+D(\tau) n,
$$


where

$$
\begin{aligned}
& C(\tau)=\left[\begin{array}{cc}
0 & 1 \\
\frac{0.72}{x_{2}-6} & -0.72 \frac{x_{1}-10}{\left(x_{2}-6\right)^{2}}
\end{array}\right] \\
& D(\tau)=\left[\begin{array}{cc}
0 & 0 \\
\frac{0.72}{x_{2}-6} & 0
\end{array}\right]
\end{aligned}
$$

and

$$
n(\tau)=\left[\begin{array}{c}
n_{1}(t) \\
0
\end{array}\right]
$$

The expected values of $z_{1}$ and $z_{2}$ as well as their variances of the system (55) are given by

and

$$
m_{j}(t)=E\left[z_{j}(t)\right]=\phi_{1 j}(t) z_{j}(0), \quad j=1,2
$$

$$
\lambda_{r s}(t)=2\left[\sum_{k, t=1}^{2} \phi_{k r}(t) \phi_{l s}(t) \int_{0}^{t} f_{22}(t) \psi_{2 k}(t) \psi_{2 l}(t) \mathrm{d} t\right] \quad r, s=1,2
$$

To generate the elements of $\psi$, the computer set-up is shown in Fig. 1 with the functions $\psi_{i k}$ shown in Fig. 2. The generation of the matrix $\Phi$ is indicated in Fig. 3 with the results shown in Fig. 4.

Using a noise generator with an average spectral density of $5.28 \mathrm{~V}^{2} / \mathrm{cps}$. The theoretical values of the means and variance of the system (54) are plotted, respectively, in Figs. 5 and 6.

In order to check the theoretical means as well as the variances of the system (54), the random noise $n_{1}$ is applied to the system (54) as indicated in Fig. 3. The experiment is repeated for 100 times with a time interval of $10 \mathrm{sec}$ for each run. From the date obtained by the Sanborn recorder the means and variances can be calculated according to the following formulas.

$$
\begin{gathered}
m_{j}(t) \cong \sum_{i=1}^{100} \frac{z_{j}^{i}(t)}{100} \quad j=1,2 \\
\lambda_{j k}(t) \cong \sum_{i=1}^{100} \frac{\left[z_{j}^{i}(t)-m_{j}(t)\right]\left[z_{k}^{i}(t)-m_{k}(t)\right]}{100}
\end{gathered}
$$




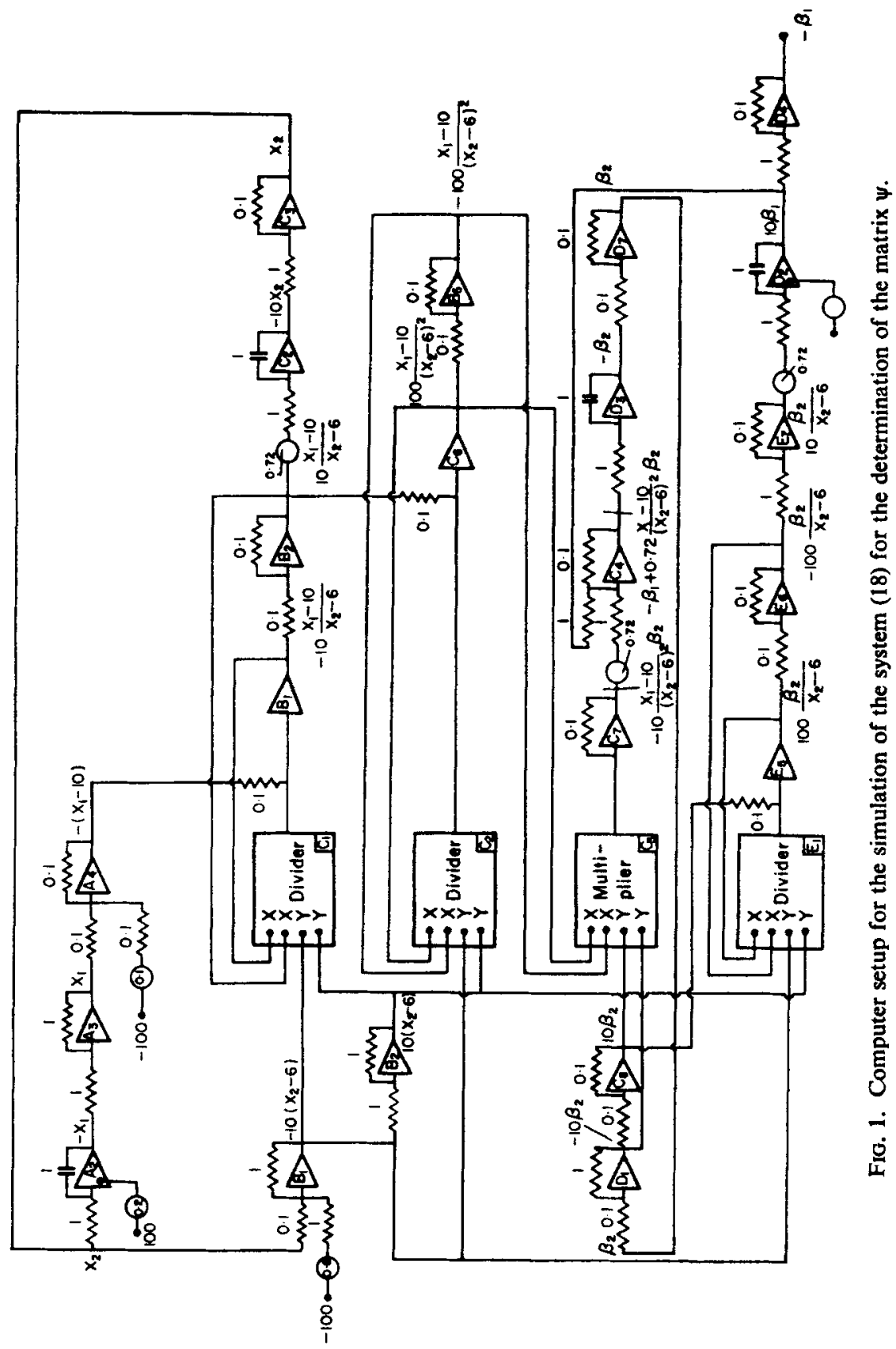



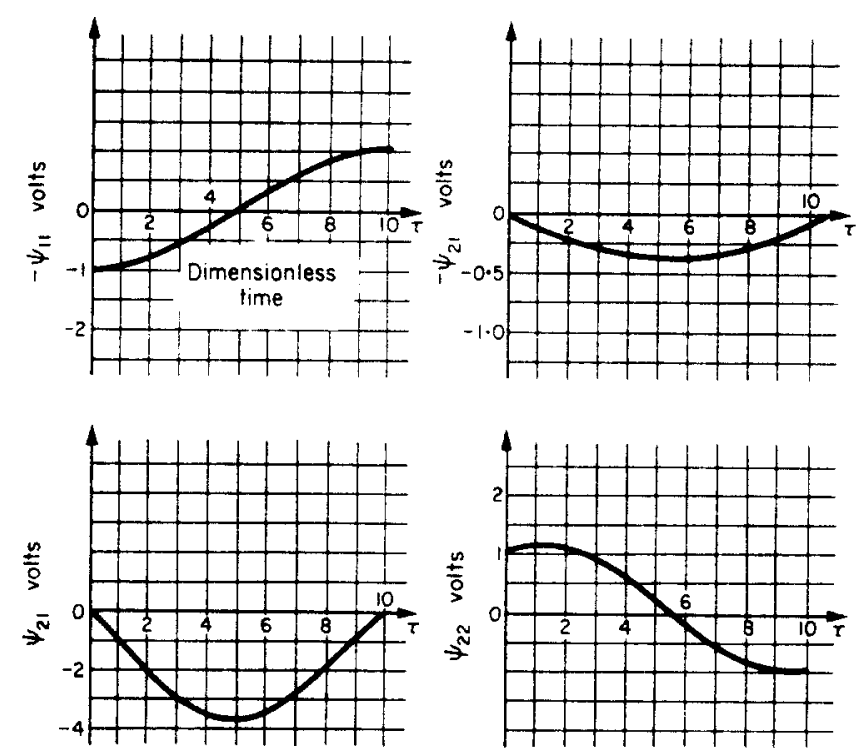

Fig. 2. Experimental determination of the eiements of the matrix $\psi$.

where the superscript $i$ indicates the $i^{\text {th }}$ run of the experiment. The results corresponding to several time instants are also plotted in Figs. 5 and 6.

It is found from Figs. 5 and 6 that the experimental results are in close agreement with theory. However, it should be pointed out that the method of confidence intervals cannot be used to compare the experimental and theoretical curves. This is due to the fact that the confidence intervals are exceedingly small and in fact that they are well within the limits of experimental errors of the measuring instruments.

\section{CONCLUSIONS AND REMARKS}

In this paper, the fundamental solution of the Fokker-Planck partial differential equation arisen from stochastic systems is obtained by means of the combination of the method of Fourier transform and the method of characteristics. This combinational method is distinct from the other methods $[7,8]$ used in solving stochastic optimal control problems in that the solution obtained by this method does not use the fact that the response of a linear system subject to Gaussian random input is also Gaussian. In view of this fact our method may be considered as a new proof of the Gaussian Invariant property of linear systems.

The concept of state for deterministic systems is well known. As a matter of fact, the concept was originated in the time of development of classical mechanics. However, for stochastic systems, the concept of state is still unsettled. Recently, Stratonovich [9] of the U.S.S.R. introduced the probability density function as the state of a stochastic system.

[7] W. M. Wonham: Stochastic problems in optimal control, R.I.A.S., Report 63-14. May (1963).

[8] J. J. FLorentine: Optimal Control of Continuous Time, Markov, Stochastic Systems.

[9] R. L. Stratonovich: Conditional Markov processes, Theory Probab. Applic. 5, No. 2 (1960) 


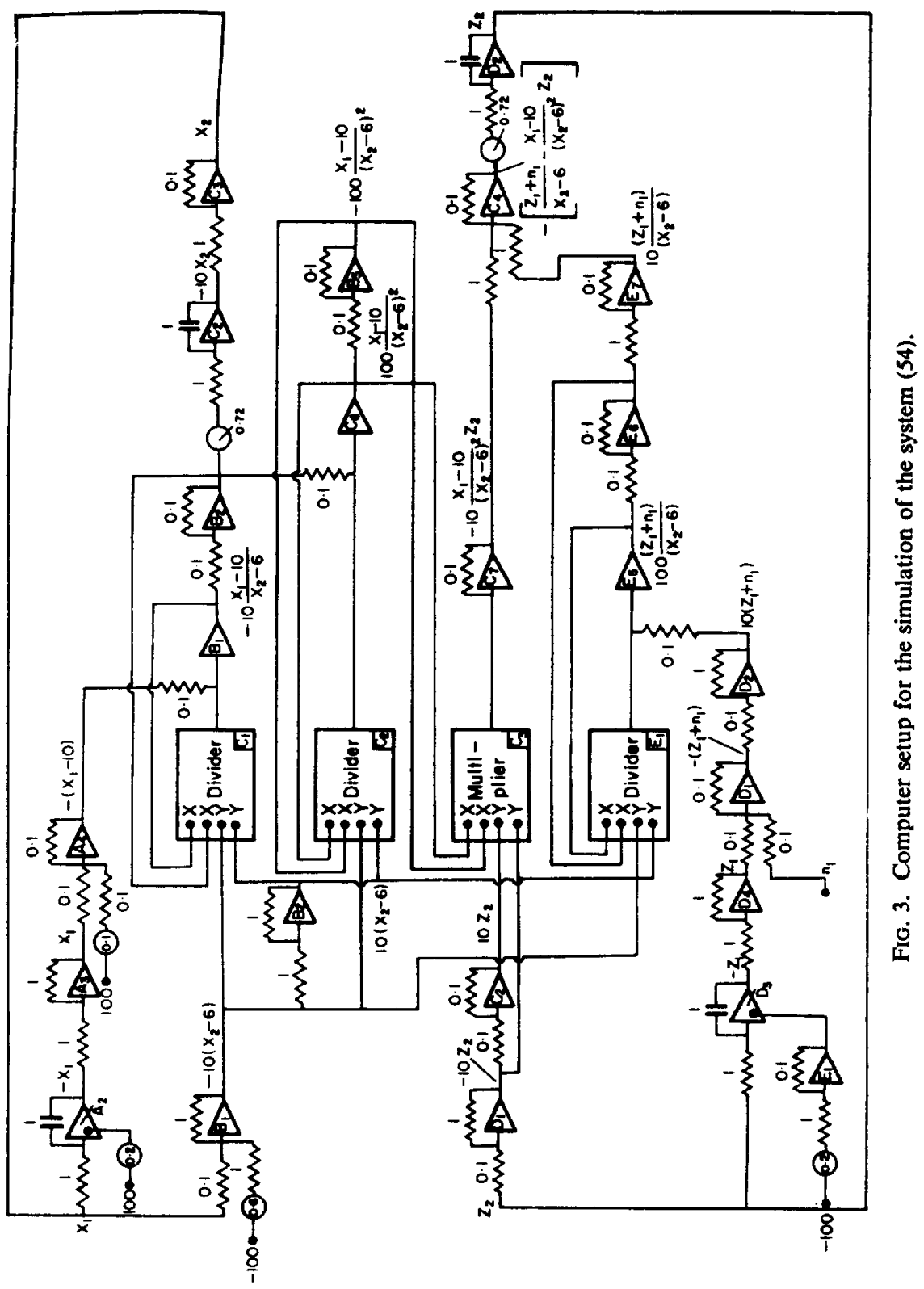



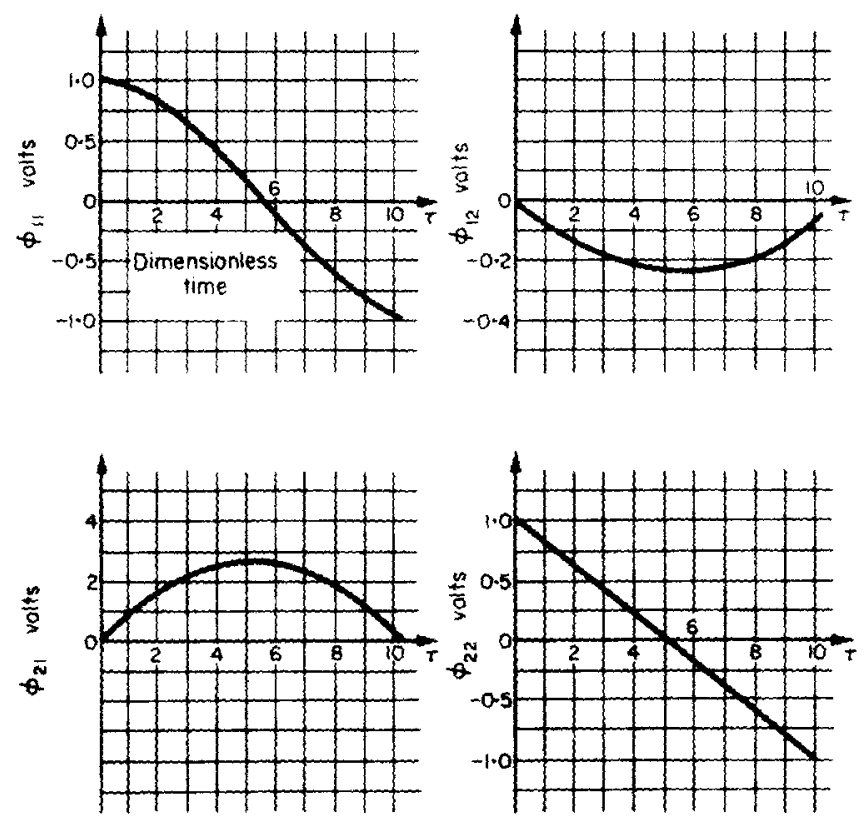

FIG. 4. Experimental determination of the matrix $\Phi$.
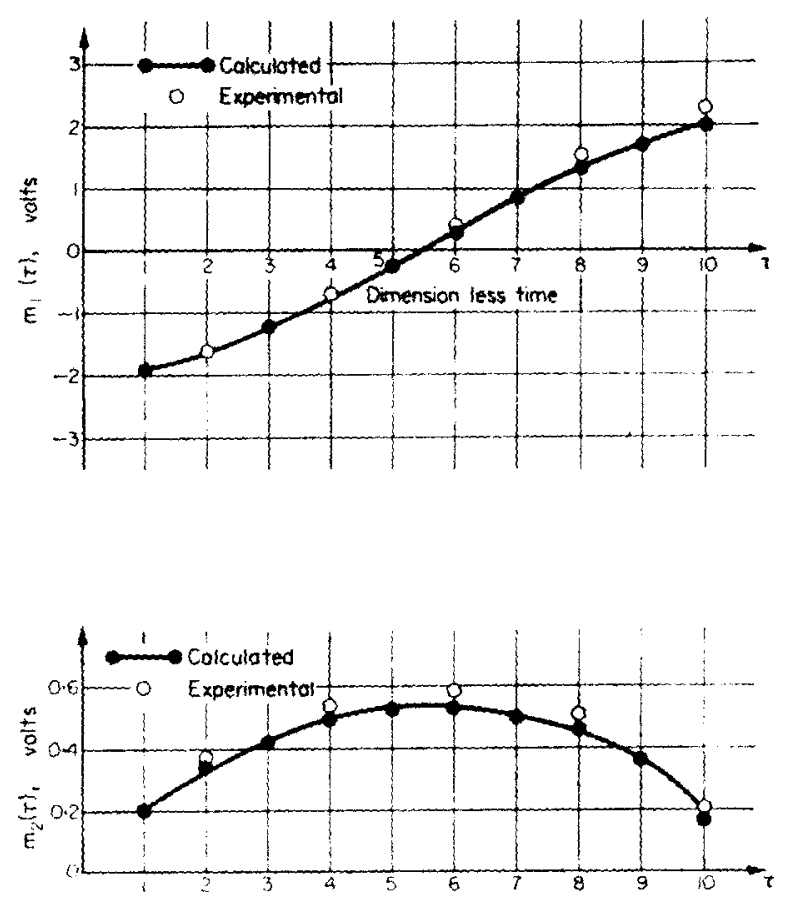

Fig. 5. The means of the system (54) as a function of dimensionless time $\tau$. 

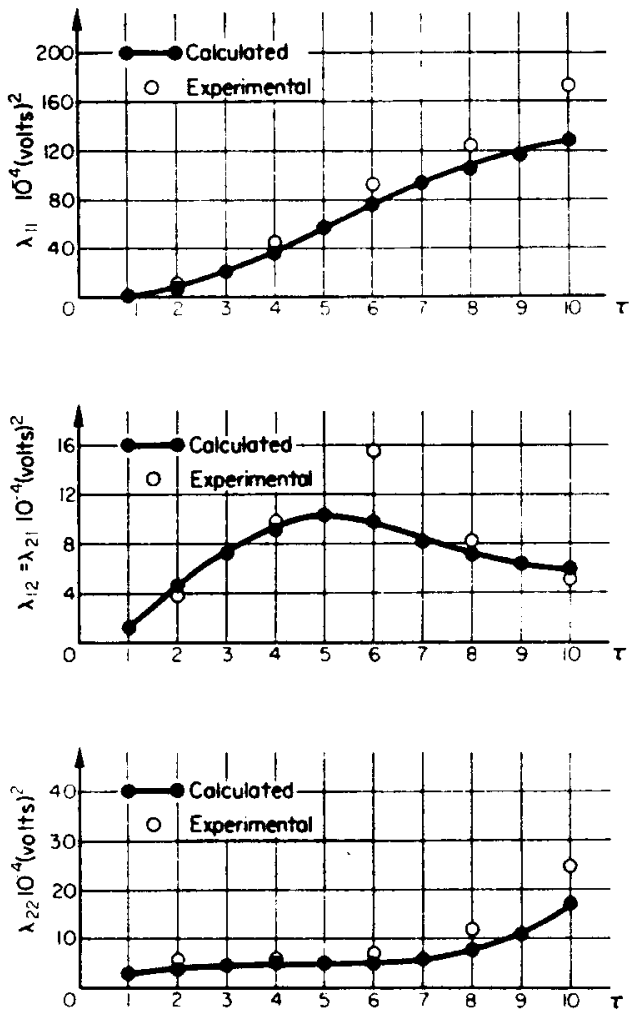

FIG. 6. The variance of the system (54) as a function of dimensionless time $t$.

This idea of using probability density function as the state of a stochastic system was mentioned by Bellman [10]. The improved version of StRatonovich's work on the concept of state was presented by KUSHNER [10, 11] and WONHAM [12]. The key concept of state is the separability of the past from the future, consequently the concept of the state for a stochastic system is equivalent to the method of finding the minimum set of variables such that by using this set of variables the system can be described as a Markov process. In this paper, we have shown that for a linear system subject to a stochastic input described by a set of linear differential equations excited by white noise, the state of the system may be defined as the original deterministic state of the system adjoined by the deterministic state of the set of differential equations describing the stochastic input to the system. The above concept of augmented state has been discussed by several authors; however, a specific definition of state to a particular stochastic system such as ours, as far as we know, has not been given.

[10] R. Bellman: Adaptive Control Processes, Princeton University Press (1961).

[11] R. KUSHNER: On the dynamical equations of conditional probability density functions, with applications to optimal stochastic control theory, J. math. Analysis Applic. 8, 332 (1964).

[12] W. M. Wonham: Some applications of stochastic differential equations to optimal nonlinear filtering, R.I.A.S., Report (1964). 
In current control literature modeling theorems for stochastic systems often have been proved only mathematically, experimental verifications of the theorems are usually bypassed. However, it is well known unless the topics under theortical study are purely theoretical in nature such as controllability and observability, the success of a modeling theorem must be conformed with the experimental data. Simply for this reason, our analytical results were checked with our simulated experimental data, even though the experiment may be crude in nature.

Résumé-Le présent article se rapporte à l'analyse stochastique de systèmes décrits par des équations différentielles linéaires avec des perturbations aléatoires, qui interviennent souvent dans l'étude du comportement variationnel d'un système de commande optimale le long de sa trajectoire nominale, en raison de perturbations aléatoires dans les paramètres de l'installation ou d'erreurs de mesure dans les variables d'état.

Le vecteur aléatoire peut être un vecteur de bruit blanc ou peut être engendré par des équations différentielles excitées par un bruit blanc. Le résultat général, obtenu au moyen de l'équation de Fokker-Planck, ne revèle pas seulement la proprieté de stabilité du système mais permet également de déterminer stochastiquement l'état du système à chaque instant. Une vérification experimentale est constituée par la simulation d'un système du second ordre sur un caiculateur analogique et le résultat trouvé est conforme à la théorie.

Zusammenfassung-Die Arbeit befasst sich mit einer stochastischen Anaiyse von Systemen, die durch lineare Differentialgleichungen beschrieben werden und stochastischen Störungen unterworfen sind. Eine solche Problemstellung tritt bei der Untersuchung des Verhaltens eines zeitoptimalen Regelungssystems entlag seiner Nennphasenbahn bei Anwesenheit von zufälligen Schwankungen der Regelstreckenparameter und von Fehlern bei der Messung der Zustandsvariablen auf.

Der zufällige Vektor kann ein Vektor von weissem Rauschen sein oder durch eine mit weissem Rauschen gestörte Differentialgleichung erzeugt werden. Unter Benutzung der Fokker-Planck-Gleichung zeigt das allgemeine Ergebnis nicht nur die Stabilitätseigenschaften des Systems, sondern gestattet auch, für jeden Zeitpunkt die Bestimmung des Zustandes des Systems in stochastischem Sinne. Die experimentelle Nachprüfung durch Simulation eines Systems zweiter Ordnung auf einem Analogrechner zeigt ein mit der Theorie übereinstimmendes Ergebnis.

\footnotetext{
Абстракт-Настоящая статья относится х вероятностному анализу систем описываемых линейными дифференциальными уравнениямп с случайными помехами, которые часто встречаются прн нзученин вариационного поведния системы оптимаљьного управления вдоль ее номинальной траекторин, из за случанных помех в параметрах о6'скта или-же из за измеритеьных погрешностей в координатах состояния.

Случайный вектор может быть вектором белого тума кли-же может быть создан дифереренциальными уравнениями возбухденными бельм шумом. Общий результат, полученный путем уравнения Фоккера-Планка, только обнарухивает свокство устойчивости системы но и позволяет такхе определить вероятностное состоянне системы в каждый момент времени. Экспериментальная проверка состоит в моделировании системы второго порядка на аналоговой вычислительной машине и найденный результат соответствует теории.
} 